\title{
Key Technologies for Safe and Autonomous Drones
}

\author{
Mahmoud Hussein ${ }^{1}$, Réda Nouacer ${ }^{1}$, Federico Corradi ${ }^{2}$, \\ Yassine Ouhammou ${ }^{3}$, Eugenio Villar ${ }^{4}$, Carlo Tieri $^{5}$, and Rodrigo Castiñeira ${ }^{6}$ \\ ${ }^{1}$ Université Paris-Saclay, CEA, LIST, Software and System Engineering Department (DILS), F-91120, Palaiseau, France \\ ${ }^{2}$ Ultra Low Power Systems for IoT, Stichting IMEC Nederland, Eindhoven, The Netherlands \\ ${ }^{3}$ LIAS/ISAE-ENSMA, Futuroscope, France \\ ${ }^{4}$ Microelectronics Engineering Group, TEISA Dpt. University of Cantabria Santander, Spain \\ ${ }^{5}$ TEKNE S.R.L, Province of Chieti, Italy \\ ${ }^{6}$ INDRA SISTEMAS SA (INDRA), Calle Villalar, 3, 28001 Madrid, Spain
}

\author{
mahmoud.hussein@cea.fr,reda.nouacer@cea.fr,federico.corradi@imec.nl, \\ yassine.ouhammou@ensma.fr,villar@teisa.unican.es,c.tieri@tekne.it,and rcastineira@indra.es
}

\begin{abstract}
Drones/UAVs are able to perform air operations that are very difficult to be performed by manned aircrafts. In addition, drones' usage brings significant economic savings and environmental benefits, while reducing risks to human life. In this paper, we present key technologies that enable development of drone systems. The technologies are identified based on the usages of drones (driven by COMP4DRONES project use cases). These technologies are grouped into four categories: U-space capabilities, system functions, payloads, and tools. Also, we present the contributions of the COMP4DRONES project to improve existing technologies. These contributions aim to ease drones' customization, and enable their safe operation.
\end{abstract}

Keywords- Drones/UAVs, U-space, System Functions, Tools, Architecture, Trusted Communications, Safe-decision.

This paper is an extended version of "Mahmoud Hussein, Réda Nouacer, Yassine Ouhammou, Eugenio Villar, Federico Corradi, Carlo Tieri, and Rodrigo Castiñeira, Key Enabling Technologies for Drones, the EUROMICRO Digital System Design Conference (DSD 2020), August 26 - 28, 2020, Portorož, Slovenia.".

\section{INTRODUCTION}

Unmanned Aircraft Vehicles (UAVs), which referred to as drones commonly, are air vehicles and associated equipment that fly autonomously or remotely controlled, where it does not carry a human operator [1]. Drones are increasingly used in commercial and civilian applications such as surveillance, monitoring constructions, agriculture, etc. They can perform air operations/missions that are difficult for execution by manned aircrafts. In addition, their usage brings significant economic saving and environmental benefits while reducing the risk to human life. Recently, drones have already made the leap to the leisure market, and reached millions of sales. Therefore, evolution on technology, regulations and society acceptance is in favour of an accelerated deployment of drones for professional applications. According to a study performed by the Teal Group [2], the world civil drone production is forecasted to make up to $\$ 73.5$ billion in the next decade, rising from $\$ 2.8$ billion worldwide in 2017 to $\$ 11.8$ billion in 2026 (i.e., a $15.5 \%$ annual growth rate in constant dollars).

The UAV market already has a great variety of hardware, software, and operational products to offer. However, the key element for global UAV success story is not just defined by these aspects as it is largely depends on local regulation. The international regulatory bodies work together in the context of JARUS (Joint Authorities for Rulemaking on Unmanned Systems) initiative. In addition to national authorities, Europe participates in this work through EASA (European Aviation Safety Agency). As a result, there have been a recent promising developments in Europe with the publication of: procedures for the operation of unmanned aircrafts [3], regulation on unmanned aircraft systems and on thirdcountry operators of unmanned aircraft systems [4], and Vertical Take-off and Landing (VTOL) certification [5].

The integration of UAVs into non-separated airspace requires essential technologies. The technologies exist but are not yet mature enough for cross-regional implementation due to missing technical standards. Thus, this paper aims to identify essential/key enabling technologies that are required to develop a safe and a customizable drone system. The drone system includes the drone itself, the ground control station, and the communication between them. The drone (UAV) is composed of three main parts: (a) the platform that includes structure, engines, servomotors, etc. (b) the avionics is formed by all the electronic systems and peripherals that allow the UAV's flight: the communications link, the flight controller, and the navigation system; (c) the payload is made by one or several sensors needed to carry out the UAV's mission.

To identify the key enabling technologies, we first identify the drone usages based on the COMP4DRONES's project use cases. The use cases cover five domains: (a) application of drones to optimize transport operations, and infrastructure management; (b) smart application of drones for digitalization of a construction process's state, and analysis of underground constructions' status; (c) logistic using heterogeneous drones fleet; (d) drone and wheeled robotic systems for inspection, surveillance and rescue operations with enhanced navigation and autonomous abilities; (e) smart and precision agriculture. Based on the different use cases, two groups of drone usages are identified: flying stages (e.g., takeoff, cruise, land, etc.), and mission specific operations (e.g., survey a land, check a crop health, inspect offshore infrastructures, etc.).

Second, a general architecture (structure) for the drone's system which is then used by a process to identify the different components/technologies of the system is presented. Our architecture separates the different concerns of the system into three main layers: control, flight management, and planning. Therefore, each layer can be easily customized separately without affecting the other architecture layers.

Third, based on the list of drone usages, state of the art drone systems, and best practices, we have identified the different components in the three architectural layers. These

(C) 2021. This manuscript version is made available under the CC-BY-NC-ND 4.0 license http://creativecommons.org/licenses/by-nc$\mathrm{nd} / 4.0 /$ 
components are then generalized to define the key enabling technologies. The technologies are divided into three groups: system functions (e.g. flight control, positioning, coordination, etc.), payloads (camera, lidar, etc.), and tools (system design, data analytics, mission planning, etc.). Also, there are technologies needed to support the $U$-space services. These services enable secure, safe, and efficient access to airspace by the drones (e.g., geofencing, security, telemetry, etc.) [6].

Finally, improvements of the COMP4DRONES project to existing technologies (in order to enable ease customization of drones' systems and their safe operation) are discussed. These improvements are grouped into four categories: integrated modular reference architecture, safe autonomous decisions, trusted communication, and minimization of the system's design and verification effort.

The remaining of this paper is organized as follows. Section II presents use-cases of the project and the unified list of drone usages derived from them. In Section III, we present the process for identifying the key enabling technologies for drone systems, and introduce the technologies. The project contributions are discussed in Section IV. Finally, conclusions are put forward in Section V.



Drones for optimization of transport control, operation and infrastructure management



Drones for virtual design, construction and operation of transport infrastructures

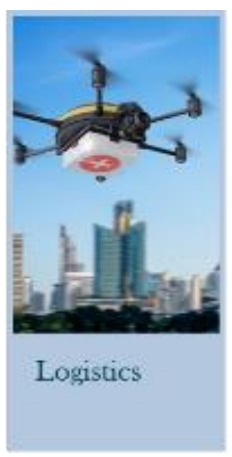

Logistics using heterogeneous drone fleets



Drone and wheeled robotic systems for inspection, surveillance and rescue operations



Smart precision agriculture: from drone to rover
Figure 1: Use-cases driving the key enabling technologies
In the following, we give a brief description of the different demonstrators in each use case.

\section{1)Transport}

This use case demonstrates the technology developed in COMP4DRONES within the transport domain. It uses drones as sensors and monitoring devices for different transport infrastructures. Three demonstrators are deployed in this use case: traffic management, port operations, and railway infrastructure.

First, the demonstrator for traffic management focuses on the deployment of drones as monitoring devices of the road traffic conditions, and the detection and early response to incidents. To do so, drone facilities incorporate capabilities to request a drone's flight over an area of the infrastructure identified by its operators. The demonstrator objective is to use drones for improving the efficiency and automation of monitoring activities as well as to lower their costs, upgrading the control and maintenance of transportation operations and their infrastructure, offering greater flexibility and stability in solutions and services.
Second, the demonstrator of port operations focuses on the deployment of a captive drone as a mobile system for security and aerial surveillance in real time in the port environment. The usage of the system aims to improve the port surveillance infrastructure, saving deployment costs of a new network of fixed cameras, and providing a surveillance system of higher quality that is accessible from any point to increase security.

Third, the demonstrator for railway infrastructure focuses on deploying flight command and control exclusively through a cellular network. In this demonstrator, a drone is used in the routine monitoring of a railway infrastructure construction and the exploitation stages.

\section{2)Construction}

New technologies allow a great digitalization of the state of construction, which in turn allows a greater control in all phases of the construction process. Thus, the main challenge of the use case is to develop the technology required to carry out any type of operation that allows the digitalization of the state of the constructive process. 
In this use-case, two demonstrators are deployed. The first demonstrator aims to develop technologies required to carry out any type of operation that allows the digitalization of the state of the constructive process of a civil Infrastructure. In this demonstrator, a section of road under construction is analyzed.

The second demonstrator focuses on the deployment of drones as tools for analyzing the underground constructions (e.g., tunnels). In this demonstrator, the drone platform obtains data in real-time, keeps the distance to wall sides, and detects any interfering obstacle inside the tunnel. During the flight, the drone platform acquires data and, as a result, the system gives high accuracy models. The information produced improves the efficiency of the activities of planning, exploring, mapping of tunnels, measurements of the underground environment, generation of complete high-precision models, and production of base models for implementation.

\section{3)Logistics}

The logistics domain could benefit from a drone system because it can provide features that the conventional solutions cannot offer such as simultaneous delivery at several locations for a reduced price, and accessibility to remote areas or areas without infrastructures. To demonstrate those capabilities, this use case develops two demonstrators.

The first demonstrator aims to deliver geophysical sensors using an autonomous fleet of UAVs in the context of METIS project. The METIS project has been started by TOTAL in 2014 to unlock hard-to-image in hard-to-access location. The project has developed an innovative 3D high-density geophysics solution that enhances the quality of subsurface images, and lowers costs and environmental footprint.

The second demonstrator aims at delivering a parcel in a hospital using droid (to carry it inside a building), and a drone (to carry it between buildings) to achieve fast delivery. The demonstrator is performed in two stages. In stage one, which is the longer, the design of the droid and the drone is done in an industrial site to show the interest of their coupling to carry light parts. The second phase aims to repeat the industrial site experiment in hospitals for transporting medicines or test samples with drones and droids.

\section{4) Surveillance and Inspection}

The main goal of this use case is the realization of drones with enhanced sensory abilities by means of sensory systems and novel control. These enhanced drones can then be safely used in indoor and outdoor environments for industrial inspection, security, surveillance, and in rescue operations. Two distinct demonstrators are being developed in the context of this use case: inspection of off-shore turbines, and mapping of a disaster site.

First, in the context of inspecting offshore turbines, the targeted innovations are: (a) detect imperfections such as corrosion deterioration of paint using a hyperspectral camera with images analytics in varying lighting conditions, and (b) real-time and robust collision avoidance in harsh environment (e.g., strong wind), and keep safe distance from the turbines.

Second, in the context of mapping of a disaster site, a fleet of multi-robots is used for navigating in and mapping of an unknown environment. This fleet consists of lightweight, small, and larger drones with processing. The focus here is on multi-drones' collaboration in a GPS (Global Positioning System) denied environment. Thus, collaborating drones have to create a common model of the environment, which includes the automatic detection of points of interest.

\section{5)Agriculture}

This use case shows the developments of the project in the field of agriculture. It is conceivable that over the next few decades the use of drones and rovers for monitoring plant growth and health, as well as for treatments and harvesting will increase. In this use case, two demonstrators are being implemented in two different areas of agriculture.

The first demonstrator is focusing on crop monitoring with emphasis on health and growth of crop. In this demonstrator, the idea is to give evidence that certain manual operations can be perfectly carried out in an autonomous manner by advanced autonomous systems. This reduces impact on the environment of certain operations (i.e., precisely sizing the amount of water and pesticides to be used and acting on spot where needed, promptly activating treatments at first symptoms on individual crops/plants), while saving human effort.

The second demonstrator is designed to assist winemakers in their work, and to minimize the workload and the travel time to remote and poorly connected to the infrastructure vineyards. Data is collected by flying autonomous over the vineyards to save considerable time, otherwise the assessment and evaluation of the plants and the soil needs to be done locally. This is faced through two key approaches: using the drone as gateway, and using the drone as an earth-observation platform.

\section{B. Unified List of Drone Usages}

Based on the project demonstrators, we have identified a unified list of usages of the drone systems. The list is divided to two categories flying stages and mission specific operations.

\section{1)Flying Stages/Operations}

A flight has several stages: taxiing, takeoff, climb, cruise, descent, and landing. If a drone completes all these flight stages, then a flight cycle is completed. First, the taxing is the move of the drone on the ground using its own power. Second, the take-off is the phase of flight in which the drone leaves the ground and becomes airborne. Third, the climb is the operation to increase the altitude of the drone. Fourth, the cruise is a flight phase that happens between a climb to an altitude and before the drone starts to descend. Fifth, a descent during a flight cycle is any portion where the drone decreases its altitude. Finally, landing is the last stage of a flight, where the drone returns to the ground.

\section{2)Mission Specific Operations}

Based on the project use cases, we have identified the specific missions that can be performed by the drones. These missions are classified into four categories: inspection, aerial surveillance, indoor missions, and logistics. In the following, we list these missions.

\section{1) Inspection}

Inspection missions are performed for (1) checking the air quality in and around structures and buildings; (2) assessing water quality where water is important to human survival, and then water quality need to be monitored and protected; (3) inspecting off-shore infrastructure by drone to decide whether a human is needed for doing a detailed follow-up inspections; (4) checking the health of plants to effectively manage plants, soil, fertilization, and irrigation in order to treat shortages, diseases, or pests in a timely, targeted, and local manner; (5) gathering geological data for helping geophysicists in identifying and better approximating the location and presence of natural gas, minerals, and oil. 


\section{2) Aerial Surveillance}

The aerial surveillance is used for (1) performing measures over places that are hazardous or difficult to be reached on foot; (2) surveying a land to determine the three-dimensional extension or terrestrial positions of objects of interest and compute the distances/angles between them; (3) gathering video and images for spotting an activity that can be missed by standard cameras with narrow view; (4) managing traffic operation based on video streaming in real time from an accident location to the transport control center, and video analysis using artificial vision, and embedded vehicle-tracking capabilities on the drone; (5) digitalizing state of a constructive process by capturing data from areas under construction, highways, etc.; (6) measuring crops height for providing timely and reliable spatial information to farmers and decision-makers in the precision agriculture; (7) communicating with delivered seismic sensors to verify the good landing and coupling of the sensors.

\section{3) Indoor Missions}

The indoor missions are used for analyzing underground constructions status by capturing a georeferenced scanner of the progress in the construction of a tunnel, and accessing GPS denied environment autonomously to (1) explore the unknown space and create a common reference model of the environment; (2) identify points of interest (e.g., human victims), and calculate routes for humans to extract those victims using the reference model.

\section{4) Logistics}

With drones, a set of logistics mission can be executed such as (1) delivering seismic sensors to unlock many hard-toreach places where it is not worth going to shoot seismic in a traditional ground-based way; (2) delivering parcel though the coordination between a drone to fly between buildings, and a droid and to reach places inside these buildings; (3) spraying crops to save a great amount of fertilizers and human effort by automatically providing the right amount of fertilizer to every plant; (4) watering plants by UAVs equipped with sensors that can identify parts of a crop that need more water. In this way, it is possible to improve the efficiency of water use, in the right places at the right time and in the right quantity.

\section{Key ENABLING TeCHNOLOGIES}

In this section, we describe how the key technologies are identified, and present the key technologies in detail.

\section{A. Identification of Key Enabling Technologies}

To identify the key enabling technologies for drones, there is a need for a general structure/architecture of the drone systems. The idea behind this general system architecture is to have a well-defined system partitioning for easy development of the key technologies and their integration later on. The COMP4DRONES vision towards an architecture for the drone systems is shown on Figure 2 [7]. It separates the different concerns of the system into three layers: control, flight management, and planning.

The control layer contains the low-level control modes of the UAV (e.g., take-off, landing, trajectory following, etc.). The control behaviors are realized through actuators in case of they are performing an action, or using sensors in the case of perceptional tasks.

The flight management layer is responsible for selecting a pre-defined (designed or generated) plan in response to an environment situation propagated from the control layer, and cannot be handled in the control layer. It is also responsible for executing the selected plan by sending commands to the control layer to perform tasks such as fly to, scan area, etc. Furthermore, in the case of, no plan exists to cope with the environment situation, a request is sent to the planning layer to provide a new plan.



Figure 2: A layered architecture of a UAV system

The planning layer is responsible for high level planning. Its main task is to generate/provide the different plans that enable the drone to perform intended missions in an optimized way. This layer is responsible for task planning, motion planning, reasoning and diagnosis mechanisms, and execution monitoring, etc.

Missions (Scenarios / Services)

Scan a specific region with available AVs to generate a saliency map consisting of geolocations of injured civilians



Figure 3: An example of UAV different layers

An example instance of the architecture (shown in Figure 2 ) is presented in Figure 3. This instance is taken from a rescue mission, where a number of UAVs are used to scan an area to find injured civilians. To achieve this mission, a plan has to be either designed or automatically generated using a planning algorithm at the planning layer. The plan consists of a set of 
tasks such as scan area, transmit data, fly-to destination, etc. These actions are performed sequentially or done in parallel. The sequence of actions is coordinate and executed using the execution engine in the management layer. This layer also has a coordination component to enable the collaboration between different UAVs. Finally, the control layer is responsible for executing the control behaviors to realize the tasks. For example, the fly-to task is achieved through take-off, follow a trajectory, and landing control behaviors. The behaviors use hardware and software components to execute the different tasks (see Figure 3).

Based on the proposed architecture and its instance in Figure 2 and Figure 3, the missions (requirements) are the main driver for the different elements of the architecture, where the requirements are used to define the plans. These plans consist of a set of tasks, which are realized using control behaviors. These control behaviors are executed using hardware and software components as shown in Figure 4. These steps also define/require a number of tools to support the engineers in doing this process.



Figure 4: A process for identifying the key technologies

Following the process in Figure 4, the unified list of usages (requirements) discussed in Section II.B are used to identify: tasks to satisfy the requirements, control behaviors to perform the tasks, hardware/software components that provide the resources to execute the behaviors, and tools required to create the identified components (i.e. the key enabling technologies for drones). Examples of the identified technologies are shown in Figure 5. These technologies are grouped into system functions, payloads, and tools (e.g., data analytics, mission planning, and system design). The system functions are common/shared elements that can be reused across different drone systems (e.g. planning algorithms, plan execution, flight control, and communication). The payloads are specific to certain mission (application), but also can be used in more than one mission (e.g. camera, lidar, and geophysical sensors).



Figure 5: Example identified technologies
In addition to the technologies identified above, the drone systems must be enhanced with a set of $U$-space capabilities (see Figure 6). These capabilities ensure a secure, safe, and efficient access to airspace for the drones [6]. The capabilities include e-identification, geofencing, navigation, etc. In the following sections, we list and describe the identified key technologies.



Figure 6: U-Space services and the drone autonomy [6]

\section{B. Drone Capabilities for $U$-space}

The capabilities expected for enabling U-space services are shown in Figure 7. These capabilities are divided into three groups to support different types of service: foundation, initial, and advanced services [6]. First, capabilities for the foundation services include: geofencing, security, telemetry, operation management, e-identification, communication, command and control, surveillance, and navigation. Second, the initial services capabilities include tracking and emergency recovery. Third, the capabilities for the advanced services are detect and avoid, vehicle-to-infrastructure communication, and vehicleto-vehicle communication (see Figure 7).



\section{1)U1: U-space Foundation Services}

The U-Space foundation services include: (1) $e$ identification which is the ability for identifying the drone and its operator in the U-space; (2) geofencing that is the drone ability to be compliant with time, geographical, and altitude restrictions defined by the geo-fencing service; (3) security which is making the drone able to protect itself and its data (i.e. interaction with infrastructure and other vehicles) from attacks; (4) telemetry that is the ability for transmitting measurement data from a drone to another drone or to a 
service provider for meeting the demands of relevant services; (5) communication, navigation, and surveillance that makes the drone able to meet performance requirements of the communication, navigation and surveillance in the specific environment in which it will operate. This capability consists of on-board sensors and equipment (e.g. voice radio relay, data link, etc.) as means to achieve the required performance; (6) command and control which is the drone's ability to communicate with a ground control station for conducting the flight (normally through a specific data link); (7) operations management that is the ability for planning and managing the drone missions. This involves accessing and using of all relevant information for planning, notifying, and operating a mission.

\section{2)U2: U-space Initial Services}

The initial services enable the tracking of the drone by providing flight parameters that include at least its position and altitude, and emergency recovery which is the ability of drones to take into account failure modes such as link failure, command and control (C2) failure. It also takes measures for ensuring the safety of the vehicle itself, other vehicles, and property and people on ground.

\section{3)U3: U-space Advanced Services}

The advanced services of the U-space are (1) vehicle to infrastructure communication (V2I) which is the drones' ability for sharing information with infrastructure components; (2) vehicle to vehicle communication $(V 2 V)$ that is making drones able to communicate information to each other. The nature of the exchanged information and its performance depend on the application; (3) detect and avoid which is ability of drones to detect hazards, or cooperative and non-cooperative conflicting traffic, and to take the appropriate actions to comply with the applicable rules of flight.

\section{System Functions}

The drone system functions are the core functions required for the drone to perform its flying stages in safe and efficient manner. The different system functions are shown in Figure 8 and are described in the following.

\section{1)Flight Control}

The control of the drone during its flight is performed through intelligent mission management and intelligent outloop control. First, the intelligent mission management (IMM) is onboard and/or ground technologies for providing a desired mixture of human-directed and autonomous drone operations [8]. It enables the shift of the human role in conducting drone missions from vehicle operators to be users and requesters for drone applications. It increases the autonomy level in performing complex UAV operations.

Second, the intelligent outer-loop control (IOLC) is an onboard capability to enable autonomous and semi-autonomous operations. Traditional control systems such as autopilots or flight management systems (FMS) achieve navigation and guidance goals defined by human through controlling the vehicle flight surfaces [9]. IOLC achieves high-level mission goals through extending the traditional approach. For example, a flight management system can be tasked to make the aircraft follows a specified route, and an IOLC can be tasked with a broader goal such as monitoring a set of ground targets for events of interest to alert users whenever such events occur. For meeting such goals, the system should be able to control not only vehicle surfaces, but also its communications, sensor payload, and other sub-systems.

\section{2)Flight Navigation}

To perform waypoints navigation of the drone from a source to destination, a number of functions are needed. These functions include flight planning and scheduling, fail-safe mission, contingency management, deconfliction, and detect and avoid.

First, the flight planning and scheduling are general technologies that take higher-level goals, constraints, and objectives and then turns these into detailed plans and schedules, that can be performed by humans or drones [10]. The difference between planning and scheduling is that: (a) planning includes more choices about what objectives to achieve, and the different actions needed to achieve them; (b) scheduling involves activities that are given, and the main decisions is to order these activities, and assign resources to them. Both scheduling and planning are cross cutting technologies and have a wide application in many areas of intelligent systems.

Second, the fail-safe mission is the ability of a UAV system to adapt to software or hardware failures for having an acceptable level of safety. This function is among the most critical ones in drones [11]. Such technology is generic to any drone application for providing high reliability and it is one of the important features needed to access the air space. Reports regarding drones indicated that they are looking for "reliability comparable to a piloted aircraft".

Third, the contingency management is an on-board capability for reacting to unforeseen events. It is particularly needed to minimize the likelihood of property damage and human casualties. It also maximizes the likelihood of drone and payload survival [12]. In general, it includes a number of techniques that are designed to increase robustness of a drone in response to uncertainties. These uncertainties have many forms such as failures/degradations of hardware components (actuators, sensors, etc.), missing precise information about the drone environment (wind, visibility, cloud cover, etc.), or unknown events (volcanic eruptions, fires, etc.). In the face of these uncertainties, techniques for contingency management are useful to improve mission safety and productivity.

Fourth, the deconfliction is a function that is used to resolve potential conflicts that can occur between drones' trajectories in the phase of planning strategic trajectories [13]. This function aims to reduce the workload of the air traffic controller in conflict resolution through designing efficient trajectories with minimal number of potential conflicts. Thus, once the drone(s) is cleared for flying, the workload of controller will be more monitoring with less conflict prediction and resolution (i.e., more flights can be accommodated by the controller at a given time).

Finally, the detect and avoid (DAA) is a basic requirement for drones to safely operate. The collision avoidance process involves capturing the surrounding environment, and assessing potential of colliding with hazards that are detected. It takes corrective actions to avoid the hazards when a collision is upcoming [14]. The hazards that are of concern in collision avoidance are: ground (earth surface), drones (other vehicles in the space), weather, and obstacles (e.g., power lines, towers, ground equipment, etc.). The pilot's eyes can be used to visually detect and track the hazards. However, when the operator of the drone is remotely located, an automatic DAA system is foreseen as an important feature to allow the drone operation with an equivalent level of safety of a piloted drone. 


\section{3)Positioning}

The drone position is an important aspect to make the drone flight safe. The position can be estimated by an indoor positioning system, or by simultaneous localization and mapping algorithm. It also used to preserve the geo-fence, and tag images with their coordinates.

First, the indoor positioning system (IPS) is a set of devices used for locating people or objects when GPS and satellite technologies fail or lack precision. This usually occurs in multistory buildings and underground locations [15]. A set of techniques and devices can be used for providing indoor positioning ranging from (a) reconfigured devices such as Bluetooth antennas and $\mathrm{Wi}-\mathrm{Fi}$, smart phones, digital cameras, and clocks to (b) purpose-built installations with beacons and relays placed throughout a defined space. IPS has many applications in military, commercial, and inventory tracking industries.

Second, the simultaneous localization and mapping (SLAM) is the process of recording environment and location awareness in a map of an autonomous vehicle [18]. SLAM is an important component in self-driving cars and other autonomous robots to enabling awareness of their location and best routes to their destination. Through the creation of its own maps, SLAM provides a quicker, a more autonomous and an adaptable response than the pre-defined routes.

Third, the geofencing is a virtual barrier that can be created by combining GPS network and LRFID (Local Radio Frequency Identifier) connections (e.g., Wi-Fi, Bluetooth, etc.). This boundary is forced by the drone during its flight [16]. Such technology is available since many years with an early adaption to monitor cattle with the help of GPS for providing alerts when livestock left its predefined boundaries. There is also other uses such as monitoring of fleet vehicles to provide early warning when anything abnormal occurs.

Fourth, the geo-referencing is the task of assigning realworld coordinates to the pixels of a raster [17]. Such coordinates are obtained through performing field surveys (i.e., collect coordinates by a GPS device for easily identifiable features in the map or image). In some situations, when looking for digitizing scanned maps, the coordinates can be obtained from the markings on the map image itself. Using such sample coordinates, the image can be warped and fitted within a chosen coordinate system.



Figure 8: Drone system functions

\section{4) System and Environment Status}

The system and its environment status are needed to execute the mission safely and take decisions in the right time. Such status is provided through data fusion and intelligent system health management. First, the data fusion is the task of integrating many data sources to produce consistent, useful, and accurate information that cannot be provided by any individual source of data [19]. The drone telemetry system has a limited bandwidth. This bandwidth must be allocated between the flight control function and the payload elements. Thus, it is not possible to transmit all data out of drone, and then data processing is needed for reducing the volume of transmitted data. This enables real-time data analyses, as well as ensuring some level of data capture in the event the loss of the platform. Second, intelligent system health management (ISHM) is a technology designed for assessing a system's health and recommending (performing) actions that ensure it will remain healthy in future [20]. This technology contributes to drone safe operations in several ways such as recovering from faults, and recommending actions in the presence of other faults. ISHM techniques can be either on the drone or on the ground.

\section{5)Coordination}

To execute a mission, a collaboration between different vehicles is needed. These vehicles can aerial or ground. First, the necessity of coordination between unmanned aerial vehicles (UAV) and unmanned ground vehicles (UGV) is particularly evident to do missions in remote areas, where human may be exposed to dangerous situations [21]. In these situations, monitoring and exploration missions could be performed safely by robots where they can easily gather information from the environment safely. Multiple UAVs and UGVs are certainly able to play preprogramed missions by 
moving around in certain scenarios. But, the most interesting challenge is providing them with a decisional autonomy and opportunity for cooperation and adaptation according to realtime situations with little human intervention.

Second, the swarm formation and cooperation are the reasoning and making decision entity that is responsible for the use of mission requirements, observations (by the UAV itself and other UAVs in the fleet), and system constraints to have a specific organization of the UAVs [22]. In brief, it needs to compute trajectories of the different UAVs and make decisions on how tasks are allocated for achieving a good team behaviour. Coordination means achieving and sustaining good formations or task distribution between drones in a selforganizing manner. The coordination can be done at a global or a local level depending on the mission specification and the drones' capabilities.

\section{6)Communication}

Communication is an important aspect in a drone system to enable the exchange of data between the different parts of the system. Such communication can be ether net-centric or over the horizon communication. First, the net-centric communications is a concept of operation that uses advanced technology for shifting to a data-centric paradigm from an application-centric paradigm [23]. It allows the users to access applications and services by web services. This concept increases situational awareness and robustness of missions via networking sensors, decision making, and faster command and control.

Second, over the horizon communications (OTH), which is commonly referred to BVLOS (Beyond Visual Line of Sight) communications, is a basic function that is required for UAVs to operate in the global airspace [24]. OTH is needed for Command and Control (C2Link), status and health of the vehicle, situational awareness, and real (near real) time vehicle position (longitude, latitude, and elevation above the surface of the earth at a given time) using the GPS or the UAV's onboard navigation system. There is also a need to have OTH with UAV payload to receive real-time data, snapshots, or determine status of on-board data recorders.

\section{Payload Technologies}

The drone system includes a set of payload technologies to support the drone's mission specific operations. The payload technologies include optical sensors, microwave sensors, insitu sensors, and external sensors as shown in Figure 9.

\section{1)Optical Sensors}

The optical sensors are either active or passive. First, the active optical remote sensors (i.e., lidar) use optical source such as a laser for sensing targets. The targets can be either hard objects (e.g., other vehicles, terrain, and obstacles) or the atmosphere through scattering light from molecules and aerosols [25]. Measurements of hard target are useful for geographical information systems, and for payload delivery. On the other hand, atmospheric parameters can be measured such as gas concentration, aerosol density, wind, and cloud cover. The advantage of lidar-based approaches is that spatial and temporal resolution is much higher than the other sensory systems.

Second, the passive optical sensors are the major imaging devices that are found on aircraft and satellites. The devices essentially capture infrared radiation emissions, and direct or reflected solar energy. They project them to photosensitive detectors through an imaging optics system [26]. However, there also non-imaging sensors that are used to collect radiometric and/or spectral data from a single point. Such sensors are typically used for measuring radiations from the earth and the sun, and are used to characterize the intervening atmosphere. Both types of the sensors are appropriate within the UAVs systems.



Figure 9: Payload Technologies

\section{2)Microwave Sensors}

There are two types of microwave sensors: active and passive. First, the active microwave sensor is an imaging radar. It emits microwave radiation. The sensor then records the echoes returned from the scene to be observed [27]. This system contains wavelengths varies from less than a centimeter to three meters that depend on the application. To achieve a good resolution, the system transmits a waveform with a bandwidth that depends on the desired resolution, then many pulses are collected and combined by signal processing approaches to achieve the desired resolution. Second, the passive microwave sensors are used for both surface imaging and atmospheric measurements. They gather data through detecting light, vibrations, radiation, etc. [27]. A main challenge for such sensors is the required spatial resolution. For example, Cold Land Processes may need spatial resolutions of a hundred meter at microwave frequencies, while spatial resolution of one $\mathrm{km}$ is needed for Soil Moisture measurements which is very challenging.

\section{3)In-situ Sensors}

The in-situ sensors include chemical sensor arrays, meteorological data, difference frequency generation (DFG) lasers, and $\mathrm{CO}_{2}$ detection.

First, the chemical sensor arrays technology allows measurements for a range of chemical species in the UAV's surrounding environment [28]. Such type of micro-sensors are small and consume less power in comparison with the standard instrumentation. Thus, they can be easily integrated with drone hardware and software systems. Second, meteorological data such as air density, temperature, and wind affecting UAV operations need to be measured [28]. For example, both pressure and temperature need to be measured for determining the air speed and its accuracy. 
Third, the difference frequency generation (DFG) lasers are used as advanced in-situ detectors for tracing gases and their composition. Also, they can be combined with enhanced absorption spectroscopy [28]. DFG-based sensors that allow measurements of gas traces are small and non-cryogenic. Such measurements are an important element of meteorological or atmospheric research missions. Fourth, the $\mathrm{CO}_{2}$ Detection sensor measures $\mathrm{CO}_{2}$ by a quantum cascade laser spectrometry in the flight configuration [28]. The $\mathrm{CO}_{2}$ detector uses a laser spectrometer in a flight configuration for measuring $\mathrm{CO}_{2}$ with long term precision of $0.05 \mathrm{ppm}$ and with $0.1 \mathrm{ppm}$ as an absolute accuracy (i.e., matching the requirement for most of atmospheric-based drone mission).

\section{4)External sensors}

To execute different types of missions, a set of external sensors are required such as dropsonde, seismic, weather, and perimeter.

First, the dropsonde is a weather reconnaissance device that is designed to be dropped from an aircraft. There are four basic measurements that are performed by the dropsonde: temperature, pressure, winds, and humidity [29]. Measurement of temperature, humidity, and pressure are generally performed using a thin-film polymer and thermistor package, while winds are typically measured by GPS receivers (either true or codeless GPS). Second, the seismic (geophysical) sensors are deployed on the ground to record soundwave velocities coming from an activated seismic source (like vibrator truck). Number and spatial sampling of these sensors will drive the quality and fidelity of the final image [30]. In general, seismic sensors are cabled-based system that requires heavy ground logistic to put them in place. With the technology advances, seismic sensors can now be delivered from air using drone swarms.

Third, the weather station is very useful to monitor the changes in real time. It allows the UAV system to detect and act when important changes in the forecast happen [31]. For example, the drone could be ordered to finish its task and return without any further take-off, or if the change came quickly, it can be ordered to urgently return to the base. Fourth, the perimeter sensors use light-detecting, passive infrared (PIR), and infrared (IR) sensors to monitor surroundings. When a movement is detected by the sensors, notifications are sent to the security system [32]. Such sensors can be hidden among plants or placed in the open area to create an undetectable, invisible protective barrier.

\section{E. Tools}

To support the development of drone systems, a number of tools need to be developed. These tools are divided into two main groups: tools for service specification, and tools for system development (see Figure 10).

\section{1) Service Specification}

The tools in the service specification group are used for specifying the user requirements, acceptance testing, data analytics, and mission planning.

First, the user requirements referred to as needs specify what the user wants from the system (i.e., what activities the system enables the users to do) [33]. These requirements are documented generally in a user requirement document as narrative text. The requirements are signed by the user. Then, they are used as the main input to create the system requirements. Second, the acceptance testing is a level of testing, where a system is tested for user acceptability [34]. The purpose of this type of test is to evaluate compliance of the system with different business requirements and evaluate whether it can be delivered or not. It is also considered as a formal testing for the achievement of user requirements and needs. To determine whether a system satisfies acceptance criteria or not, business processes is conducted. The processes enable the user/customers to accept the system or not.

Third, the data analytics is a process of cleaning, inspecting, transforming, and modeling data with the aim to discover useful information and conclusions to support decision-making [35]. Data analytics has many facets and diverse techniques, which used in different science and business domains. It plays an important role to make decisions more scientific and to help businesses to operate in an efficient way. Example data analytics technique is data mining that focuses on knowledge discovery and statistical modelling for predictive purposes. Another technique is the business intelligence that relies on aggregation with a main focus on business information. Fourth, mission planning is the process to produce a flight plan that describes a proposed drone flight. It has two safety-critical aspects: compliance with air traffic requirements to avoid collisions, and fuel calculation to ensure that the drone can safely reach the desired destination [36]. It also minimizes flight cost by choosing route, speed, and height that minimizes the necessary fuel. The produced flight plan is used by the Air Traffic Services for aircraft tracking, and finding a lost aircraft in search and rescue scenarios.



Figure 10: Tools for drone systems

\section{2) HW/SW System Development Cycle}

To enable the development of the drone systems hardware and software, the tools should support system's requirements specification, design, implementation, integration, validation and verification.

First, the system requirements are the main blocks that developers use for building the system [37]. These requirements are statements that explain what the system should do. They are classified as either functional (i.e., specify something that is required by the users to perform their tasks) or non-functional (i.e., certain system qualities) requirements. Second, the system design process provides the sufficient detailed information about the system and its sub-system to 
enable the system implementation [37]. The result of the design process is views and models of the system architecture.

Third, the system implementation follows the structure created during the system design, and the system analysis results to construct system components. These components must meet stakeholders and system requirements specified in early phases of the system development [37]. Implementation phases yield the lowest-level elements of the system. The elements are bought, made, or reused. This phase also involves processes for hardware fabrication and software realization.

Fourth, the system integration is the process to bring together the different component/sub-systems into one system [37]. It also ensures that the sub-systems (i.e., different software and hardware components) work together as a system. The integrated sub-systems may include computer networks, business processes, and enterprise applications.

Finally, the verification and validation $(\mathrm{V} \& \mathrm{~V})$ is checking that a system meets its specifications and fulfills its intended purpose (goals) [37]. It also refers to the software quality control and software testers are responsible for this task. Simply, software validation is does our software meet its intended goals, while software verification is does the right software has been built.

\section{COMP4DRONES CONTRIBUTIONS}

In the following, we describe the contributions of the COMP4DRONES project based on the identified key enabling technologies. The contributions are classified into four groups: integrated modular reference architecture, safe autonomous decisions, trusted communication, and minimization of the system design and verification efforts.

\section{A. Integrated Modular Reference Architecture}

The main goal of this group is to provide a reference architecture for flexible embedded platforms. This will enable easy and efficient customization of drone systems (especially the autopilot software) to meet requirements of an application domain. The software architecture of the embedded platform will be hardware-independent and have generic components which can be plugged-in easily. The components/technologies developed in the project are clustered into four categories: hardware platforms, basic software, sensing, and image/video processing. In this section, we describe these groups briefly.

Hardware Platforms. To enable the execution of a drone system, a number of hardware components are being developed in the COMP4DRONES project. Such hardware components are either to speed up the execution of the system functions in general, or they are developed for specific system feature. First, the demand for onboard computational power of modern drones is exponentially growing due to the everincreasing request for autonomous operations. To satisfy this request, a more powerful (in terms of operational throughput) computing platform must be embedded on the drone. The platform should also maintain operational constraints related to the power envelope, and interoperability and connectivity with standard drone software stacks. Therefore, in the project, a set of platforms are developed to speed up the execution of the different functions of the drone system.

Second, SLAM (Simultaneous Localization and Mapping) techniques are considered to be a mature field. But, the actual problem is the lack of a modular architecture (involving hardware and firmware configurations) that can deal with heterogeneous sensors' configurations and provide an easy-to- use, easy-to-adapt, extensible, and reusable configuration package. Thus, a technique is being developed in the project to solve this problem. Finally, most of drone missions require image and video processing which can be heavy based on the mission specification. Thus, a module for object detection and positioning is being developed in the project to enable faster execution of objects detection and their positioning.

Basic Software. To support a drone mission, a number of basic software components are needed. Thus, in the context of COMP4DRONES, generic components for mission control and power management are being developed. First, to enable safe and autonomous operations in uncertain environment, a control component that implements potential barriers is developed. It supports navigation and mission planning by providing geo-awareness, and avoiding any violation of geofencing. Second, many applications require multiple drones to work together in a cooperative manner in order to complete complex missions quickly and efficiently. Thus, a mission controller is being developed to support fleets' formation and management to enable such cooperative behavior. Finally, power supply is an important aspect to make sure the drone flight is safe. Thus, power management systems are proposed to manage the battery, and to select a trajectory with the lowest power consumption.

Sensing. The drone should be equipped with a number of sensors to perceive its status and environment. First, to capture high quality images, Hyperspectral (HSI) cameras are being investigated for capturing, processing, and storing images of a good quality. Second, knowing the drone position is very critical for a mission execution, where the position should be available during the whole flight in different environments (i.e., in both indoor and outdoor environments). Thus, an ultrawideband-based indoor positioning and simultaneous localization and mapping algorithms are being developed in the project and used for localizing the drone in the indoor situations, while an outdoor position and attitude estimation provides accurate position of the drone in the outdoors.

Image/Video Analytics. Most of drone missions require data analysis. Thus, in the project, a set of components are developed to support image and video analysis. Such analysis is drone through conventional neural network (CNN), highdynamic-range imaging (HDR) tone mapping, hyperspectral imaging (HSI) pipeline, artificial intelligence algorithms, and video content analysis algorithms.

\section{B. Safe Autonomous Decisions}

Command, control, communication, computing, and artificial intelligence capabilities at the edge are required to enable autonomous navigation and commercially viable business opportunities. This group focuses on technologies for enhancing mission-critical functions, while increasing the drones' autonomy to enable safe and continuous operation. It follows a modular approach in which many components are developed to serve the use-cases envisioned in the project. The modular approach eases the integration and customization of the drone's platform for the various use cases.

To enable the (semi-)autonomous operations, the standard Guidance, Navigation, and Control (GNC) framework is used. This framework is extended with newly designed systems and subsystems/technologies of task-specific responsibility. Each technology has a detailed interface description, functionality, and application-specific programming interfaces. In the following, we describe these technologies. 
Payload Technologies. A number of sensing technologies are investigated and developed to enhance sensory perception of the drones. Such sensing technologies include hyperspectral imaging, event-based sensing, position tracking technologies, and sensory fusion strategies.

System-on-Chip (SoC) Technologies. A large portion of the development is devoted to enable advanced functions on SoC technologies, which contain standard processors and microcontrollers (ARM/RISC-V) in conjunction with fieldreprogrammable-gate arrays (FPGA). The SoC platforms are industry-rated technologies that enable the acceleration of real-time data processing, sensory fusion, and advanced machine vision tasks (i.e., object detection, optic flow, obstacle avoidance, etc.). The flexibility provided by these technologies enables the change of electrical functionalities at runtime. Drones equipped with $\mathrm{SoC}$ allows fast integration of advanced functionalities while enabling efficient acceleration of the computational expensive machine-learning workloads. Thus, several application-specific accelerators are being developed by the COMP4DRONES consortium for some of the most compute-intensive and challenging tasks. These tasks include object detection, visual analytics, simultaneouslocalization and mapping, etc.

Technologies for Real-time Mission Control. An equally important aspect addressed is the creation of interfaces and control stations for flight management, monitoring, mission real-time visualization, geofencing technologies, and real-time control. Particular focus is devoted for the development of flight management systems that can be integrated with the Unmanned Traffic Management system (UTM).

Runtime Safety Technologies. Runtime safety systems are a fundamental pillar. To evaluate various risk scenarios in uncertain environments, the project proposed a run-time safety mechanism and a framework. In the proposed framework, the runtime functionality of the drone is ensured by monitoring the execution of predefined invariants, and potential system interventions for improving system safety are suggested.

\section{Trusted Communication}

The trust in the communication pursued by the project means enlarging the conditions under which communications continue to function regarding two main aspects: the physical availability of the link, and accidental or malicious attacks to which the system is subjected.

The first aspect is tackled by components grouped under the name "Robust Multi-Radio", which addresses multilink capabilities to support fleet coordination, indoor navigation, payload data retrieval, vehicles monitoring and management. The second aspect has two specializations: (1) addressing security risks before an attack occurs, and (2) defending while an attack is occurring. The attacks are tackled/prevented by the "Security Management" components that principally address the cryptography from the hardware up to the protocols. On the other hand, when attacks occurred, their signs are detected and reactions are done by "Reactive Security" components. These components are applied to data transmission and Global Navigation Satellite System (GNSS) that employ algorithmic and Artificial Intelligence (AI) techniques. In the following, we describe the components' groups.

Robust Multi-Radio Communications. To enable robust multi-radio communication, a number of components are being developed in the project. These components offer the following solutions: integration of commercial off-the-shelf link technologies such as IEEE 802.11 and LTE, bandwidth aggregation and store-and-forward on the different available links, support for a fleet of UAVs, communication among Ultra-Wideband (UWB) nodes (fixed on the UAV) that support the positioning for indoor navigation, adaptation of the video coding and compression to the link bandwidth, an edge gateway to interface the entities participating in the network, and a cloud gateway for monitoring and management.

Security Management. To improve the security of the data transmission, a number of components are being developed. These components include, first, a hardware component which is a chip that supports the Transport Layer Security (TLS). It hardens the security aspects of pure software-based systems that are prone to hacking and identity spoofing attacks. Second, a software component that on one side enhances existing security protocols with novel features such as forward secure key exchange integration into TLS 1.3 for low-latency, and integration of post-quantum cryptographic primitives for long-term security. On the other side, it incorporates novel types of protocols such as the use of anonymous credentials for authentication with strong identity privacy. Third, a component that considers IoT applications (fixed sensors and a drone that is used as gateway). Fourth, a security framework to identify and to correct issues that may suppose a threat to drone-to-drone and drone-to-infrastructure communications.

Reactive Security. The reactive security components have Introduction Detection System (IDS) capabilities to detect and mitigate security attacks to data transmissions or to Global Navigation Satellite System (GNSS). The components also provide other functionalities such as encryption and decryption, help in establishing countermeasures, provision of reliable and accurate navigation data through GNSS, and other on-board sensors data fusion.

\section{Minimization of the System Design and Verification}

The objective of this group is to define and set-up a system engineering framework and development workbench adapted to drone applications. This platform covers all facets of the Vmodel, including modelling, optimization, code generation, performance analysis, verification and validation, etc. The proposed workflow, integrating design, performance analysis and verification tools supports incremental certification by the separation of concerns and reuse of qualified components. In the following, we describe specific contributions to existing tools.

Drone system modelling and code generation. The use of system modeling languages and tools for designing cyber physical systems (CPSs) has experienced significant growth in recent years. This trend has led to several studies using model-based design for CPSs, especially in COMP4DRONES scenarios for UAVs. For example, a model-based approach and tools are being developed in the project for (a) designing drones that are explicitly efficient to perform a variety of tasks, (b) implementing drone software system starting from an abstract representation of the system, and (c) supporting the validation of drone systems. In addition, the continuous demand for high performance drone systems has led system designers to use heterogeneous components based on FPGA technologies, and then selecting a suitable implementation is problematic due to the large number of heterogeneous HW /SW components on the market. In this respect, tools in the project are developed to consider the impact that mapping to the HW platform would have on the system behavior, and 
derive the coarse-grained reconfigurable co-processing units used for defining and integrating application-specific HW into an overlay compute cluster.

Drone systems validation and verification. The verification and validation of Guidance, Navigation, and Control (GNC) algorithms is fundamental for the development of autonomous drones. Thus, in the context of the project, modeling and simulation tools are used to reduce verification and validation costs by testing these algorithms on virtual test benches before deploying them on physical prototypes. For example, path planning, a subpart of GNC, is critical in the drones' development. It uses algorithms to determine optimal trajectories to guide a drone on its mission. Thus, tools to determine and validated a trajectory against a mission's requirements are developed. In addition, tools are being implemented for battery recharging and replacement operations, ensuring communication security, and consistency checking of system specification.

Drone system analysis and optimization. Starting from the early stages of a drone systems' design cycle, analysis and optimization are performed at high level to effectively explore the design space and provide relevant information to support design decisions. Thus, first, to help drone manufacturers iterate effectively during the initial design phase, a multi-level simulation framework for drone-based services is being developed.

Second, as the design becomes more refined (i.e., the drone analysis and optimization activities involve physical systems and their performance) high fidelity models are needed to enable trade-offs between different drones' architectures (based on performance metrics). The models are also used as a virtual test bench to support the continuous development and verification of GNC algorithms. For these purposes, software tools dedicated to modelling and simulation of dynamic and multi-physics systems are utilized. Furthermore, a simulation tooling for hardware-in-the-loop testing of drone subsystems and components is provided. It allows faster development iterations, and the application of agile methodologies on drone hardware development, especially towards the development of firmware and embedded software.

Finally, for autonomous drones that operate in closed environments, their reliability depends on the capacity to determine their location without relying on GPS. Therefore, to develop effective and reliable indoor positioning systems, modelling and simulation tools are being developed.

\section{CONCLUSION}

To enable the development of safe and customizable drones, in this paper, we have identified the key technologies that are needed. To identify such key technologies, we, first, presented a summary of the different use cases in the project. The use cases cover five domains: transport, construction, inspection and surveillance, and smart agriculture. These use cases are then analyzed to identify the unified list of drones usages. The usages are classified into two groups: flying stages and mission specific operations. The flying stages include take-off, cruise, etc., while mission operations are check crop health, inspect offshore infrastructures, etc.

Second, we presented a general architecture/structure for the drone systems. This architecture is then used by a process to identify the different key technologies. This architecture separates the different concerns of the system into three layers (i.e., flight control, management, and planning).
Third, based on the identified drone usages, state of the art drone systems, and the best practices, we identified the key enabling technologies of the COMP4DRONES framework. These technologies are $U$-space capabilities (e.g. geofencing, security, and telemetry, etc.), system functions (e.g. flight control, positioning, coordination, etc.), payloads (camera, lidar, etc.), and tools (system design, data analytics, mission planning, etc.).

Finally, the COMP4DRONES contributions to improve state-of-the-art technologies are described. The improvements are in four groups: integrated modular reference architecture, safe autonomous decisions, trusted communication, and minimization of the system design and verification effort.

\section{ACKNOWLEDGEMENT}

We cordially thank all our colleagues from the COMP4DRONES project for their contributions, inspiring discussions, and fruitful collaborations. The partners cover important geographical areas in Europe (see Figure 11), and ensure that project outcomes will have lasting impact on European Drones Technology Industry.

This project has received funding from the ECSEL Joint Undertaking (JU) under grant agreement No 826610. The JU receives support from the European Union's Horizon 2020 research and innovation programme and Spain, Austria, Belgium, Czech Republic, France, Italy, Latvia, Netherlands. The total project budget is 28,590,748.75 EUR (excluding ESIF partners), while the requested grant is 7,983,731.61 EUR to ECSEL JU, and 8,874,523.84 EUR of National and ESIF Funding. The project has been started on $1^{\text {st }}$ October 2019.



Figure 11: COMP4DRONES partners

\section{REFERENCES}

[1] SESAR JU, "European ATM Master Plan: Roadmap for the safe integration of drones into all classes of airspace," 2017. [Online]. Available: https://www.sesarju.eu/sites/default/files/documents/reports/E uropean\%20ATM\%20Master\%20Plan\%20Drone\%20roadma p.pdf. 
[2] McKinsey analysis; Teal Group, "World Civil Unmanned Aerial Systems Market Profile \& Forecast," 2017.

[3] "Commission Implementing Regulation (EU)," [Online]. Available: https://eur-lex.europa.eu/eli/reg_impl/2019/947/oj. [Accessed 02 May 2020].

[4] "Commission Delegated Regulation (EU)," [Online]. Available: https://eur-lex.europa.eu/eli/reg_del/2019/945/oj. [Accessed 02 May 2020].

[5] "Special Condition for VTOL," [Online]. Available: https://www.easa.europa.eu/document-library/productcertification-consultations/special-condition-vtol. [Accessed 02 May 2020].

[6] SESAR-JU, "SESAR U-Space," 2018. [Online]. Available: https://www.sesarju.eu/U-Space.

[7] M. Hussein and R. Nouacer, "Towards an Architecture for Customizable Drones," in COMPSAC 2020 - IEEE Computer Society Signature Conference on Computers, Software and Applications, Madrid, Spain, 2020.

[8] D. Sullivan et al., "Intelligent mission management for uninhabited aerial vehicles," Remote sensing applications of the global positioning system, vol. 5661, 2004.

[9] Min, Byoung-Mun, et al., "Auto-landing guidance system design for smart UAV," International Journal of Aeronautical and Space Sciences, vol. 7, no. 1, pp. 118-128, 2006.

[10] Kim, Seon Jin, Gino J. Lim, and Jaeyoung Cho. , "Drone flight scheduling under uncertainty on battery duration and air temperature," Computers \& Industrial Engineering, vol. 117, pp. 291-302, 2018.

[11] [Fabiani, P., et al., "Autonomous flight and navigation of VTOL UAVs: from autonomy demonstrations to out-of-sight flights," Aerospace Science and Technology, vol. 11, no. 2, pp. 183-193, 2007.

[12] [Pastor, Enric, et al., "In-flight contingency management for unmanned aerial vehicles," Journal of Aerospace Computing, Information, and Communication, vol. 9, no. 4, pp. 144-160, 2012.

[13] Rahmani, Amirreza, et al. , "Multiple UAV deconfliction via navigation functions," AIAA Guidance, Navigation and Control Conference and Exhibit, 2008.

[14] Finn, Anthony, and Stephen Franklin, "Acoustic sense \& avoid for UAV's," in 2011 Seventh International Conference on Intelligent Sensors, Sensor Networks and Information Processing, 2011

[15] Saab, Samer S., and Zahi S. Nakad, "A standalone RFID indoor positioning system using passive tags," IEEE Transactions on Industrial Electronics , vol. 58, no. 5, pp. 1961-1970, 2010.

[16] Dasu, Tamraparni, Yaron Kanza, and Divesh Srivastava, "Geofences in the sky: herding drones with blockchains and 5G," in 26th ACM SIGSPATIAL International Conference on Advances in Geographic Information Systems.

[17] Turner, Darren, Arko Lucieer, and Luke Wallace, "Direct georeferencing of ultrahigh-resolution UAV imagery," IEEE Transactions on Geoscience and Remote Sensing, vol. 52, no. 5, pp. 2738-2745, 2013.

[18] Durrant-Whyte, Hugh, and Tim Bailey, "Simultaneous localization and mapping: part I," IEEE robotics \& automation magazine, vol. 13, no. 2, pp. 99-110, 2006.

[19] Waltz, Edward, and James Llinas, Multisensor data fusion, Boston: Vol. 685 : Artech house, 1990.
[20] Torens, Christoph, et al. , "Towards intelligent system health management using runtime monitoring," in AIAA Information Systems-AIAA Infotech@ Aerospace, 2017.

[21] Saska, Martin, et al., "Coordination and navigation of heterogeneous UAVs-UGVs teams localized by a hawk-eye approach," in IEEE/RSJ International Conference on Intelligent Robots and Systems, 2012.

[22] Bürkle, Axel, Florian Segor, and Matthias Kollmann, "Towards autonomous micro uav swarms," Journal of intelligent \& robotic systems, vol. 61, no. 1, pp. 339-353, 2011.

[23] Elston, Jack, et al, "Net-centric communication and control for a heterogeneous unmanned aircraft system," Journal of intelligent and Robotic Systems, vol. 56, no. 1, pp. 199-232, 2009.

[24] Mu, Yawei, et al., "Over-the-Horizon Communications System for UAVs based on Intelligent Antennas," in XXXI Simposium Nacional de la Unión Científica Internacional de Radio, 2016.

[25] Besl, Paul J. , "Active optical range imaging sensors," Advances in machine vision. Springer, New York, NY, pp. 163, 1989.

[26] Khosravi, Mohammad Reza, et al., "A novel fake color scheme based on depth protection for MR passive/optical sensors," in 2nd International Conference on Knowledge-Based Engineering and Innovation (KBEI), 2015 .

[27] Ulaby, Fawwaz Tayssir, Richard K. Moore, and Adrian K. Fung, "Microwave remote sensing: Active and passive," in Volume 3-From theory to applications, 1986.

[28] Vojinović, V., J. M. S. Cabral, and L. P. Fonseca, " "Real-time bioprocess monitoring: Part I: In situ sensors," Sensors and Actuators B: Chemical , vol. 114, no. 2, pp. 1083-1091, 2006.

[29] Weissmann, Martin, et al., "The influence of assimilating dropsonde data on typhoon track and midlatitude forecasts," Monthly Weather Review, vol. 139, no. 3, pp. 908-920, 2011.

[30] Sudarshan, Srikanth KV, et al. , "Seismic surveying with dronemounted geophones," in IEEE International Conference on Automation Science and Engineering (CASE), 2016.

[31] Rhudy, Matthew B., et al. , "UAV attitude, heading, and wind estimation using GPS/INS and an air data system," in AIAA guidance, navigation, and control (GNC) conference, 2013.

[32] Suciu, George, et al., "Sensors fusion approach using UAVs and body sensors," in World Conference on Information Systems and Technologies, 2018.

[33] Courage, Catherine, and Kathy Baxter, Understanding your users: A practical guide to user requirements methods, tools, and techniques, Gulf Professional Publishing, 2005.

[34] Miller, Roy, and Christopher T. Collins, Acceptance testing, Proc. XPUniverse 238, 2001.

[35] Elgendy, Nada, and Ahmed Elragal, "'Big data analytics: a literature review paper," in Industrial Conference on Data Mining, 2014.

[36] Evers, Lanah, et al., "Robust UAV mission planning," Annals of Operations Research, vol. 222, no. 1, pp. 293-315, 2014.

[37] Hilderman, Vance, and Tony Baghi, Avionics certification: a complete guide to DO-178 (software), DO-254 (hardware). 2007, Avionics Communications, 2007. 